\title{
Zika virus infection in a pregnant Canadian traveler with congenital fetal malformations noted by ultrasonography at 14-weeks gestation
}

Kevin L. Schwartz ${ }^{1,2,3}$, Tiffany Chan $^{4}$, Nanky Rai ${ }^{5}$, Kellie E. Murphy ${ }^{6,7}$, Wendy Whittle ${ }^{6,7}$, Michael A. Drebot ${ }^{8}$, Jonathan Gubbay ${ }^{1,4,9}$ and Andrea K. Boggild ${ }^{1,4,10^{*}}$

\begin{abstract}
Background: Following emergence of Zika virus in the Americas, a devastating new congenital syndrome has been documented, leading to significant morbidity among Zika-infected fetuses and neonates.

Case presentation: A 29-year-old pregnant woman infected with Zika virus at 9-weeks gestation in Trinidad presented with one-month of fever, headache, and myalgia with persistent viremia. Significant fetal abnormalities were identified at 14-week ultrasound, which is the earliest ultrasound to describe a severely affected fetus following Zika virus infection to our knowledge.

Conclusions: We discuss the implications of prolonged maternal viremia and the spectrum of congenital Zika syndrome detectable by fetal ultrasound.
\end{abstract}

Keywords: Arbovirus, Congenital infections, Fetal sonography, Pregnancy and travel, Zika virus

\section{Background}

Following reports of associations between congenital microcephaly and Zika virus (ZIKV) infection in pregnancy, the World Health Organization (WHO) declared ZIKV a Public Health Emergency of International Concern. ZIKV was first described over 70 years ago with limited spread outside Africa and no description of congenital abnormalities. There had been no newly discovered congenital infectious disease since cytomegalovirus and rubella in the mid-twentieth century $[1,2]$.

ZIKV is primarily transmitted through the bite of the day biting Aedes species of mosquito. Symptoms most commonly associated with ZIKV infection include diffuse or focal rash, conjunctivitis, fever, myalgia, and arthralgia [3]. Approximately $80 \%$ of ZIKV infections are asymptomatic [4].

\footnotetext{
* Correspondence: andrea.boggild@utoronto.ca

'Public Health Ontario, Toronto, Canada

${ }^{4}$ Division of Infectious Diseases, Department of Medicine, University of

Toronto, Toronto, Canada

Full list of author information is available at the end of the article
}

Identification of this emerging arboviral congenital infection has led to justifiable concern among patients, physicians, and public health officials. In the absence of a licensed vaccine and specific treatment, the focus has been on prevention and calls for enhanced understanding of the epidemic. We report a case of a pregnant woman infected with ZIKV during the first trimester with prolonged viremia and significant fetal neurological compromise identified at 14-weeks gestation.

\section{Case presentation}

A 29-year-old pregnant woman from Canada, gravida 4 para 3, developed a low-grade fever and a generalized rash at 9-weeks gestation while visiting friends and relatives in Trinidad. Symptom onset occurred 6-weeks after arriving in Trinidad and all symptoms lasted for 4 days, followed by approximately one month of fever, myalgia, and retro-orbital headaches. Seven days after symptom onset she sought medical care in Trinidad with her 4-year-old child who was experiencing similar symptoms. Her blood tested negative in Trinidad by real-time 
reverse transcription polymerase chain reaction (PCR) for dengue and chikungunya viruses, but was positive for ZIKV. An ultrasonographic examination was not performed locally in Trinidad. One month after symptom onset she returned to Canada for further evaluation.

The patient was evaluated in our centre at 13 weeks 6 days gestation, which corresponded to one month postsymptom onset. Past medical history was significant for mild cerebral palsy and scoliosis. Her only medication was a prenatal multivitamin. She denied substance use during pregnancy. On examination, temperature was $38.1{ }^{\circ} \mathrm{C}$. Heart rate and blood pressure were normal. There was no conjunctivitis, lymphadenopathy, or organomegaly. Cardiorespiratory exam was normal. The previously mentioned diffuse rash had resolved.

Blood was drawn, and at 30 days post-symptom onset, ZIKV PCR, performed by Public Health Ontario Laboratory (PHOL), was positive at a cycle threshold (CT) of 31.85 using a commercial assay [RealStar ${ }^{\oplus}$ Zika Virus RT-PCR Kit (Altona Diagnostics, Hamburg, Germany). Urine ZIKV PCR was negative by the same assay. Dengue IgM and IgG were reactive, and chikungunya IgM and IgG were non-reactive. ZIKV IgM ELISA and plaque reduction neutralization test (PRNT) serology was performed at Canada's National Microbiology Laboratory (NML) as previously described [5]. The ZIKV IgM ELISA was positive, however, the PRNT was inconclusive. Similar neutralization titres were demonstrated for both ZIKV and dengue virus (ZIKV titer 320, dengue virus titer 160) and were consistent with a previous exposure to a non-ZIKV flavivirus.

Fetal ultrasound performed at 14-weeks gestation revealed a single live intrauterine pregnancy with bilateral fluid collections in the fetal neck likely representing dilated jugular lymph sacs. The larger collection was 6-mm in size. Abnormal fetal intracranial anatomy was identified. There was asymmetry in the appearance of the lateral cerebral ventricles (Figure 1), the right

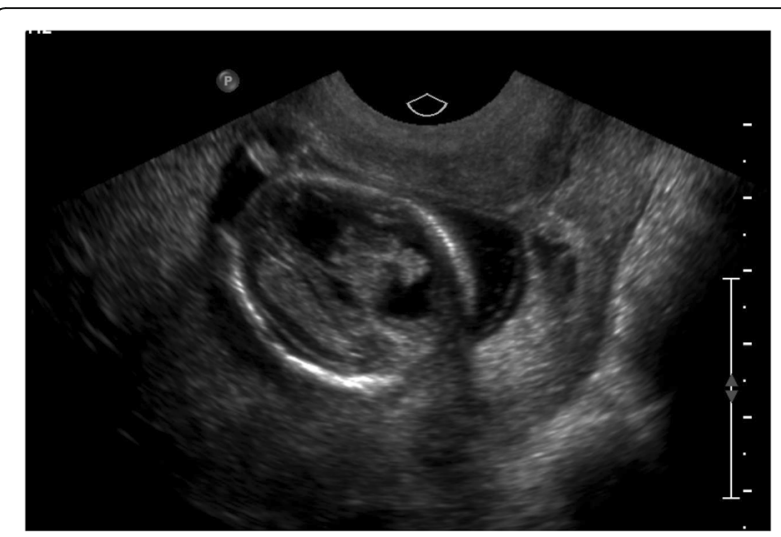

Fig. 1 Asymmetrical cerebral ventricles. Dilated right ventricle with prominent, irregular choroid plexus ventricle appeared dilated especially in the frontal horn, and the left ventricle appeared small. The left choroid plexus was prominent in the frontal horn region and the third ventricle appeared slightly dilated. Head circumference was 14.5 weeks by size. No intracranial calcifications were seen.

Therapeutic termination of the pregnancy was performed by dilation and curettage at 15-weeks gestation. Initial molecular testing of fetal tissue was performed at PHOL. ZIKV RNA was detected in the umbilical cord (CT 31.70), placenta (CT 24.89), liver (CT 24.62), and fetal neural tissue (CT 17.42) using the RealStar ${ }^{\circ}$ Zika Virus RT-PCR Kit. In addition, ZIKV sequence was confirmed in all fetal samples by RT-PCR and Sanger amplicon sequencing targeting 192 nucleotides of the ZIKV NS5 gene, based on a previously published protocol [6]. All fetal tissue specimens were forwarded to NML and retested using the CDC-designed real-time RT-PCR assay based on the ZIKV strain responsible for the 2007 outbreak in Micronesia, targeting the ZIKV envelope $(E)$ and premembrane (prM) genes [5]. ZIKV RNA was again detected in umbilical cord, placenta, and fetal neural tissue specimens. Transmission electron microscopy was performed at PHOL on placenta, umbilical cord, and neural tissue specimens, however no viral particles were visualized. Fetal tissue karyotyping for aneuploidy was performed as per standard practice, and the result was a normal male chromosomal microarray pattern.

\section{Discussion}

Uncertainties remain surrounding the risk of congenital Zika syndrome following maternal infection as well as the spectrum of disease. A recent report found that the proportion of birth defects among 442 pregnancies with laboratory evidence of ZIKV infection [7] was 20-times higher compared to the prevalence from three population-based birth defect surveillance programs during the 2013-2014 pre-Zika years [8]. Brain abnormalities or microcephaly were the most frequently recorded anomalies [8], but a number of case reports and case series have since emerged describing the spectrum of fetal effects of maternal Zika virus infection $[9,10]$. We present a case of first trimester maternal infection with significant fetal neurological malformations identified by a 14-week ultrasound following congenital infection at 9-weeks gestation. To our knowledge this is the earliest ultrasound to describe a severely affected fetus following Zika virus infection.

Previous reports have described microcephaly, intracranial calcifications, and other abnormalities in the 2nd and 3rd trimesters (Table 1) [11-18]. The greatest risk for microcephaly has been reported to occur with ZIKV infection during the first trimester of pregnancy (Table 1), with estimated risks of possible Zika- 
Table 1 Summary of previously reported Zika congenital syndrome fetal ultrasound abnormalities

\begin{tabular}{|c|c|c|c|c|c|}
\hline $\begin{array}{l}\text { Gestation at } \\
\text { First Abnormal } \\
\text { Ultrasound } \\
\text { (weeks) }\end{array}$ & $\begin{array}{l}\text { Study Author, } \\
\text { Year }\end{array}$ & $\begin{array}{l}\text { Country of } \\
\text { Infection }\end{array}$ & Gestation at Infection & Ultrasound Findings & Outcome \\
\hline 18 & $\begin{array}{l}\text { Sarno et al., } \\
2016 \text { [11] }\end{array}$ & Brazil & NR & $\begin{array}{l}\text { Microcephaly, hydranencephaly, intracranial } \\
\text { calcifications, destructive lesions of posterior } \\
\text { fossa. Hydrothorax, ascites, subcutaneous } \\
\text { edema }\end{array}$ & $\begin{array}{l}\text { Fetal demise at } \\
32 \text { weeks }\end{array}$ \\
\hline 19 & $\begin{array}{l}\text { Driggers et al., } \\
2016[12]\end{array}$ & $\begin{array}{l}\text { Unknown } \\
\text { (Guatemala, } \\
\text { Mexico or Belize) }\end{array}$ & 11 weeks & $\begin{array}{l}\text { Possible intraventricular haemorrhage, corpus } \\
\text { callosum agenesis }\end{array}$ & TA at 21 weeks \\
\hline 19 & $\begin{array}{l}\text { Pomar et al., } \\
2017 \text { [13] }\end{array}$ & French Guiana & First trimester & Severe microcephaly, cerebral hypoplasia & $\begin{array}{l}\text { Delivery at } \\
32 \text { weeks }\end{array}$ \\
\hline 19.3 & $\begin{array}{l}\text { Cortes et al., } \\
2018[14]\end{array}$ & Colombia & NR & $\begin{array}{l}\text { Enlarged subarachnoid space, decreased brain } \\
\text { volume, ventriculomegaly, malformations in } \\
\text { cortical development, simplified gyral pattern, } \\
\text { hypoplasic corpus callosum }\end{array}$ & Unknown \\
\hline 20 & $\begin{array}{l}\text { Pomar et al., } \\
2017 \text { [13] }\end{array}$ & French Guiana & First trimester & $\begin{array}{l}\text { Cystic dilation of the cisterna magna, cortical } \\
\text { hyperechogenicity, vermian hypoplasia, } \\
\text { pericallosal echogenicity }\end{array}$ & $\begin{array}{l}\text { Delivery at } \\
38 \text { weeks }\end{array}$ \\
\hline 21 & $\begin{array}{l}\text { Besnard et al., } \\
2016[15]\end{array}$ & French Polynesia & First trimester & $\begin{array}{l}\text { Microcephaly, parenchymal calcifications, } \\
\text { ventriculomegaly, absent corpus callosum, } \\
\text { absent cavum septi pellucidi }\end{array}$ & $\begin{array}{l}\text { TA at unknown } \\
\text { weeks }\end{array}$ \\
\hline 22 & $\begin{array}{l}\text { Pomar et al., } \\
2017 \text { [13] }\end{array}$ & French Guiana & First trimester & $\begin{array}{l}\text { Periventricular hyperechogenicity, irregular } \\
\text { ventricular walls, parenchymal and } \\
\text { periventricular calcifications }\end{array}$ & $\begin{array}{l}\text { Delivery at } \\
41 \text { weeks }\end{array}$ \\
\hline 22 & $\begin{array}{l}\text { Carvalho et al., } \\
2016[16]\end{array}$ & Brazil & 9 weeks & $\begin{array}{l}\text { Microcephaly, parenchymal and ocular } \\
\text { calcifications, Dandy Walker malformation, } \\
\text { arthrogryposis }\end{array}$ & $\begin{array}{l}\text { Fetal demise, CS } \\
\text { at } 37 \text { weeks }\end{array}$ \\
\hline 22 & $\begin{array}{l}\text { Besnard et al., } \\
2016[15]\end{array}$ & French Polynesia & First trimester & $\begin{array}{l}\text { Microcephaly, parenchymal calcifications, } \\
\text { ventriculomegaly, absent corpus callosum, } \\
\text { absent of cavum septi pellucidi, enlarged } \\
\text { pericerebral space }\end{array}$ & $\begin{array}{l}\text { TA at unknown } \\
\text { weeks }\end{array}$ \\
\hline 24 & $\begin{array}{l}\text { Carvalho et al., } \\
2016[16]\end{array}$ & Brazil & 5 weeks & $\begin{array}{l}\text { Dandy Walker malformation, fetal growth } \\
\text { restriction, arthrogryposis. }\end{array}$ & $\begin{array}{l}\text { Fetal demise, CS } \\
38.3 \text { weeks }\end{array}$ \\
\hline 25.3 & $\begin{array}{l}\text { Cortes et al., } \\
2018[14]\end{array}$ & Colombia & NR & $\begin{array}{l}\text { Enlarged subarachnoid space, decreased brain } \\
\text { volume, ventriculomegaly, malformations in } \\
\text { cortical development, simplified gyral pattern, } \\
\text { hypoplasic corpus callosum }\end{array}$ & NR \\
\hline 26.5 & $\begin{array}{l}\text { Cortes et al., } \\
2018[14]\end{array}$ & Colombia & NR & Dandy Walker cerebella malformation & NR \\
\hline 27 & $\begin{array}{l}\text { Carvalho et al., } \\
2016[16]\end{array}$ & Brazil & 11 weeks & $\begin{array}{l}\text { Microcephaly, ventriculomegaly bilaterally, } \\
\text { parenchymal calcifications, increased } \\
\text { subarachnoid space }\end{array}$ & $\begin{array}{l}\text { CS delivery of live } \\
\text { infant at } 38 \text { weeks }\end{array}$ \\
\hline 27 & $\begin{array}{l}\text { Cortes et al., } \\
2018[14]\end{array}$ & Colombia & NR & $\begin{array}{l}\text { Decreased brain volume, ventriculomegaly, } \\
\text { simplified gyral pattern, hypoplastic corpus } \\
\text { callosum }\end{array}$ & NR \\
\hline 28 & $\begin{array}{l}\text { Pomar et al., } \\
2017 \text { [13] }\end{array}$ & French Guiana & Second trimester & $\begin{array}{l}\text { Severe ventriculomegaly with irregular } \\
\text { ventricular walls, thin hypoplastic corpus } \\
\text { callosum }\end{array}$ & TA at 29 weeks \\
\hline 28 & $\begin{array}{l}\text { Carvalho et al., } \\
2016[16]\end{array}$ & Brazil & 8 weeks & $\begin{array}{l}\text { Microcephaly, ventriculomegaly, parenchymal } \\
\text { calcification, and oligohydraminios }\end{array}$ & $\begin{array}{l}\text { SVD of live infant } \\
\text { at } 39 \text { weeks }\end{array}$ \\
\hline 28 & $\begin{array}{l}\text { Carvalho et al., } \\
2016[16]\end{array}$ & Brazil & 8 weeks & $\begin{array}{l}\text { Microcephaly, ventriculomegaly, parenchymal } \\
\text { calcifications, cortical atrophy and cerebellar } \\
\text { hypoplasia. }\end{array}$ & $\begin{array}{l}\text { SVD of live infant } \\
\text { at } 39 \text { weeks }\end{array}$ \\
\hline 28 & $\begin{array}{l}\text { Carvalho et al., } \\
2016[16]\end{array}$ & Brazil & 10 weeks & $\begin{array}{l}\text { Microcephaly, left ventriculomegaly, increase } \\
\text { cisterna magna. }\end{array}$ & $\begin{array}{l}\text { CS delivery of live } \\
\text { infant at } 40 \text { weeks }\end{array}$ \\
\hline 28.2 & $\begin{array}{l}\text { Cortes et al., } \\
2018[14]\end{array}$ & Colombia & NR & $\begin{array}{l}\text { Enlarged subarachnoid space, decreased brain } \\
\text { volume, ventriculomegaly, malformations in }\end{array}$ & $N R$ \\
\hline
\end{tabular}


Table 1 Summary of previously reported Zika congenital syndrome fetal ultrasound abnormalities (Continued)

\begin{tabular}{|c|c|c|c|c|c|}
\hline $\begin{array}{l}\text { Gestation at } \\
\text { First Abnormal } \\
\text { Ultrasound }\end{array}$ & $\begin{array}{l}\text { Study Author, } \\
\text { Year }\end{array}$ & $\begin{array}{l}\text { Country of } \\
\text { Infection }\end{array}$ & Gestation at Infection & Ultrasound Findings & Outcome \\
\hline
\end{tabular}

(weeks)

cortical development, simplified gyral pattern, hypoplasic corpus callosum

\begin{tabular}{|c|c|c|c|}
\hline 29 & $\begin{array}{l}\text { Pomar et al., } \\
2017 \text { [13] }\end{array}$ & French Guiana & $\begin{array}{l}\text { Second } \\
\text { trimester }\end{array}$ \\
\hline 29 & $\begin{array}{l}\text { Carvalho et al., } \\
2016 \text { [16] }\end{array}$ & Brazil & 16 weeks \\
\hline 29 & $\begin{array}{l}\text { Mlakar et al., } \\
2016 \text { [17] }\end{array}$ & Brazil & 13 weeks \\
\hline 29.2 & $\begin{array}{l}\text { Besnard et al., } \\
2016 \text { [15] }\end{array}$ & French Polynesia & NR \\
\hline 29.2 & $\begin{array}{l}\text { Oliveira Melo } \\
\text { et al., } 2016 \text { [18] }\end{array}$ & Brazil & $N R$ \\
\hline
\end{tabular}

Lentostriatal vasculopathy

Microcephaly, periventricular calcifications, megacisterna magna, cerebellar vermis hypoplasia. Thymic and liver calcifications, fetal growth restriction

Microcephaly, parenchymal calcifications. Fetal growth restriction, placental calcifications.

Microcephaly, parenchymal calcifications, placental microcalcifications, ventriculomegaly, occipital pseudo-cyst, absent corpus callosum, absent cavum septi pellucidi, enlarged pericerebral space

Ventriculomegaly, corpus callosum not visualized, thalami not developed, thin pons and brainstem, non-homogeneous small mass seen at position of basal ganglia, lateral ventricles and fourth ventricle calcifications. Cataracts bilaterally, intraocular calcifications and one eye smaller in size than the other

Besnard et al., French Polynesia First trimester (15]

Oliveira Melo

et al., 2016 [18]

Brazil

Carvalho et al., Brazil 2016 [16]

Carvalho et al., Brazil 2016 [16]

Carvalho et al., Brazil 2016 [16]

\begin{tabular}{|c|c|c|}
\hline $\begin{array}{l}\text { Carvalho et al., } \\
2016 \text { [13] }\end{array}$ & Brazil & NR \\
\hline $\begin{array}{l}\text { Cortes et al., } \\
2018 \text { [14] }\end{array}$ & Colombia & NR \\
\hline $\begin{array}{l}\text { Cortes et al., } \\
2018 \text { [14] }\end{array}$ & Colombia & $N R$ \\
\hline $\begin{array}{l}\text { Carvalho et al., } \\
2016 \text { [16] }\end{array}$ & Brazil & NR \\
\hline $\begin{array}{l}\text { Carvalho et al., } \\
2016 \text { [16] }\end{array}$ & Brazil & NR \\
\hline $\begin{array}{l}\text { Carvalho et al., } \\
2016 \text { [16] }\end{array}$ & Brazil & 11 weeks \\
\hline $\begin{array}{l}\text { Pomar et al., } \\
2017 \text { [13] }\end{array}$ & French Guiana & $\begin{array}{l}\text { Second } \\
\text { trimester }\end{array}$ \\
\hline
\end{tabular}

Microcephaly, ventriculomegaly, occipital pseudo-cyst, enlarged pericerebral space

Brain atrophy, calcifications in frontal lobes including caudate, lentostriatal vessels and cerebellum. Corpus callosal and vermian dysgenesis, enlarged cisterna magna.

Microcephaly, parenchymal and cerebellar calcifications.

Microcephaly, parenchymal and cerebellar calcifications, brain atrophy, cerebellar hypoplasia. Right clubfoot, nuchal skin wrinkling, increase cisterna magna, fetal growth restriction.

Polyhydramnios, parenchymal and cerebellar calcifications. Hepatomegaly, hyperflexion of fingers, and hyperechogenicity of aortic valve, mitral valve and aortic root.

Microcephaly, ventriculomegaly, parenchymal calcifications

Enlarged subarachnoid space, decreased brain volume, ventriculomegaly, dysgenetic corpus callosum

Enlarged subarachnoid space, decreased brain volume, ventriculomegaly, malformations in cortical development, simplified gyral pattern, dysgenetic corpus callosum

Microcephaly, parenchymal and cerebellar calcifications, ventriculomegaly

Microcephaly. Right clubfoot, fetal growth restriction

Microcephaly

Microcephaly, callosal hypoplasia, gyration anomalies
Delivery at

39 weeks

Stillbirth, labour induced

TA at 32 weeks

TA at unknown weeks

Unknown

TA at unknown weeks

Unknown

SVD of live infant at 40 weeks

CS delivery of live infant at 39 weeks

SVD of live infant at 38 weeks

CS delivery of live infant at 39 weeks NR

NR

CS delivery of live infant at 39 weeks

Fetal demise, CS at 38 weeks

CS delivery of live infant at 40 weeks

Delivery at

39 weeks 
Table 1 Summary of previously reported Zika congenital syndrome fetal ultrasound abnormalities (Continued)

\begin{tabular}{|c|c|c|c|c|c|}
\hline $\begin{array}{l}\text { Gestation at } \\
\text { First Abnormal } \\
\text { Ultrasound } \\
\text { (weeks) }\end{array}$ & $\begin{array}{l}\text { Study Author, } \\
\text { Year }\end{array}$ & $\begin{array}{l}\text { Country of } \\
\text { Infection }\end{array}$ & Gestation at Infection & Ultrasound Findings & Outcome \\
\hline 37 & $\begin{array}{l}\text { Carvalho et al., } \\
2016[16]\end{array}$ & Brazil & 8 weeks & $\begin{array}{l}\text { Microcephaly, parenchymal and cerebellar } \\
\text { calcifications, left ventriculomegaly }\end{array}$ & $\begin{array}{l}\text { SVD of live infant } \\
\text { at } 39 \text { weeks }\end{array}$ \\
\hline 38 & $\begin{array}{l}\text { Carvalho et al., } \\
2016[16]\end{array}$ & Brazil & 10 weeks & $\begin{array}{l}\text { Microcephaly, periventricular and parenchymal } \\
\text { calcifications, ventriculomegaly. Fetal growth } \\
\text { restriction. }\end{array}$ & $\begin{array}{l}\text { CS delivery of live } \\
\text { infant at } 39 \text { weeks }\end{array}$ \\
\hline 38 & $\begin{array}{l}\text { Carvalho et al., } \\
2016[16]\end{array}$ & Brazil & 16 weeks & $\begin{array}{l}\text { Microcephaly, parenchymal and periventricular } \\
\text { calcifications, left ventriculomegaly. Fetal } \\
\text { growth restriction, oligohydramnios }\end{array}$ & $\begin{array}{l}\text { CS delivery of live } \\
\text { infant at } 39 \text { weeks }\end{array}$ \\
\hline 38 & $\begin{array}{l}\text { Carvalho et al., } \\
2016[16]\end{array}$ & Brazil & NR & $\begin{array}{l}\text { Microcephaly, parenchymal and cerebellar } \\
\text { calcifications, ventriculomegaly }\end{array}$ & $\begin{array}{l}\text { SVD of live infant } \\
\text { at } 39 \text { weeks }\end{array}$ \\
\hline 39 & $\begin{array}{l}\text { Carvalho et al., } \\
2016[16]\end{array}$ & Brazil & NR & Microcephaly, parenchymal calcifications. & $\begin{array}{l}\text { SVD of live infant } \\
\text { at } 39 \text { weeks }\end{array}$ \\
\hline NR & $\begin{array}{l}\text { Pomar et al., } \\
2017 \text { [13] }\end{array}$ & French Guiana & First trimester & $\begin{array}{l}\text { Microcephaly, cerebellar hypoplasia, } \\
\text { ventriculomegaly with irregular ventricular } \\
\text { walls, periventricular hyperechogenicity, } \\
\text { callosal hypoplasia with calcifications, } \\
\text { parenchymal califications }\end{array}$ & NR \\
\hline NR & $\begin{array}{l}\text { Pomar et al., } \\
2017 \text { [13] }\end{array}$ & French Guiana & $\begin{array}{l}\text { Second } \\
\text { trimester }\end{array}$ & Parenchymal hyperechogenicity & $\begin{array}{l}\text { Fetal demise at } \\
40 \text { weeks }\end{array}$ \\
\hline NR & $\begin{array}{l}\text { Pomar et al., } \\
2017 \text { [13] }\end{array}$ & French Guiana & First trimester & Microcephaly & $\begin{array}{l}\text { Fetal demise at } \\
19 \text { weeks }\end{array}$ \\
\hline
\end{tabular}

NR not reported, SVD spontaneous vaginal delivery, CS caesarean section, TA therapeutic abortion

associated birth defects in the first, second, and third trimesters of $8 \%, 5 \%$, and $4 \%$, respectively [19]. However, severely affected fetuses have been described after normal 2nd trimester ultrasounds, with subsequent abnormalities detected in the 3rd trimester (Table 1). Interestingly, there have also been reports of no differences in head circumference among infants born to women with possible ZIKV infection during pregnancy compared to women without infection [20]. The case presented herein describing severe neurological malformation in the early 2nd trimester contributes to our understanding of the spectrum of fetal abnormalities from maternal ZIKV infection.

We identified persistent viremia at 30-days post symptom onset in the context of prolonged symptomatology and documented fever. For most patients viremia is present for 3-5 days after symptom onset [3]. However, case reports have emerged demonstrating prolonged viremia in pregnant women [21]. One prospective cohort study found that clearance of ZIKV in serum, urine, and semen was seen in $95 \%$ of patients by 54 days, 39 days, and 81 days, respectively [22]. Furthermore urine testing in our patient was negative, conflicting with previous reports suggesting urine PCR is positive for longer than serum [23]. Detecting prolonged viremia may prove to be a valuable prognostic indicator for pregnant women. It is likely that viral detection in maternal serum represents high levels of fetal viral replication. Pregnant women may have greater difficulty clearing the virus, which may then lead to fetal infection. Further study investigating this finding is warranted.

The timing of fetal abnormalities ranges from identification at 14-weeks (this report) to post-natal diagnosis. Table 1 summarizes the spectrum of fetal ultrasound abnormalities detected. The spectrum of severity ranges from mild ocular or auditory abnormalities, to microcephaly, to severe brain or musculoskeletal malformations [24]. Additional information on congenital Zika syndrome is needed to better understand the spectrum of illness, however, 5 features have been recognized that are rarely seen with other congenital infections. These include severe microcephaly with a partially collapsed skull, thin cerebral cortices with subcortical calcifications, macular scarring with focal pigmentary retinal mottling, congenital contractures, and early hypertonia with extrapyramidal involvement [25].

Zika virus is currently endemic to most of South and Central America, the Caribbean, and recently areas of southern Florida and Texas in the United States. International guidelines have advised that pregnant women should avoid travel to areas with ongoing transmission of ZIKV [3]. Women unable to avoid travel are advised to use strict personal protective measures including lightcoloured long-sleeved clothing, insect repellent containing $20-30 \%$ DEET or $20 \%$ icaridin, stay in accommodations with screens or air-conditioning, and permethrin treated clothes and bed nets if outdoors [3]. Sexual transmission of ZIKV has also been described, but based on our current 
understanding of ZIKV epidemiology, likely represents a minor contributor to disease spread. To reduce the possibility of sexual transmission, we recommend that men and women avoid unprotected sexual activity with a male partner who has returned from an endemic area within six months, pending further understanding of the duration of transmission. Recent reports have demonstrated detectable virus in semen up to 6 months from symptom onset [26]. Women are further advised to defer conception for 2 months following travel to an area of ongoing ZIKV transmission, and for 6 months if their male partner accompanied them on travel [3].

Our current approach to diagnosis of pregnant patients exposed to ZIKV is outlined in recent guidelines published by the CDC [27]. Up-to-date testing algorithms can be accessed here: https://www.cdc.gov/zika/hc-providers/testing-for-zikavirus.html. Testing all exposed pregnant women by PCR within two weeks of symptom onset or exposure is recommended. Those negative for ZIKV by PCR should be tested for ZIKV IgM and if positive, confirmed by PRNT. Pregnant women 2-12 weeks from exposure, or symptom onset, should be tested for ZIKV IgM and if positive or equivocal should be tested for ZIKV RT-PCR. As illustrated by our case, however, viremia in women carrying congenitally infected fetuses may be prolonged, and in pregnant women who continue to be symptomatic beyond 2 weeks, ZIKV PCR may be considered.

Recommendations surrounding the management of pregnant women exposed to Zika virus will continue to evolve along with our understanding of the epidemiology and pathogenesis of disease. Expert bodies continue to recommend screening all pregnant women with travel to areas with ongoing transmission of ZIKV. Positive tests should prompt further evaluation with serial ultrasound assessments and possible amniocentesis for ZIKV RTPCR to facilitate counselling of patients.

Our report has some limitations. We identified higher levels of ZIKV in fetal neurological tissue compared to others, however, due to the dilation and curettage procedure performed, we cannot exclude potential crosscontamination of tissues.

\section{Conclusions}

Congenital ZIKV syndrome is a newly recognized complication of this arboviral infection which has spread throughout the Americas. This case report demonstrates that pregnant women can have prolonged viremia and early ultrasounds may detect significant fetal abnormalities. Human vaccine trials are currently underway, however personal protective measures to avoid mosquito bites and deferral of travel to risk areas are currently the cornerstone of prevention, and should be advocated by front line health care providers to all pregnant patients considering travel to ZIKV endemic areas.

\section{Acknowledgments}

We would like to thank the Viral Zoonosis section at the National Microbiology Laboratory for their efforts performing the microbiologic testing as well as Dr. Katherine W. Fong, MBBS, FRCPC, University of Toronto, for providing the ultrasound images.

\section{Funding}

Not applicable.

Availability of data and materials

All data are presented herein.

\section{Authors' contributions}

KLS and AKB conceived the report. All authors (KLS, NR, KEM, WW, TC, MAD, $J G, A K B)$ contributed equally to literature review, and interpretation of relevant clinical results. KLS, TC, NR, KEM, and AKB drafted the manuscript. All authors provided critical edits and approved the manuscript in its final form.

Ethics approval and consent to participate

Ethics approval for this case report was not sought as there was no human subjects study in which to participate. Patient written consent was obtained for use of her personal and clinical information in this manuscript.

Consent for publication

Patient written consent was obtained for use of her personal and clinical information in this manuscript.

Competing interests

The authors declare that they have no competing interests.

\section{Publisher's Note}

Springer Nature remains neutral with regard to jurisdictional claims in published maps and institutional affiliations.

\section{Author details}

${ }^{1}$ Public Health Ontario, Toronto, Canada. ${ }^{2}$ St. Joseph's Health Sciences Centre, Toronto, Canada. ${ }^{3}$ Dalla Lana School of Public Health, University of Toronto, Toronto, Canada. ${ }^{4}$ Division of Infectious Diseases, Department of Medicine, University of Toronto, Toronto, Canada. ${ }^{5}$ Department of Family and Community Medicine, University of Toronto, Toronto, Canada. ${ }^{6}$ Department of Obstetrics and Gynecology, University of Toronto, Toronto, Canada. ${ }^{7}$ Sinai Health System, Toronto, Canada. ${ }^{8}$ National Microbiology Laboratory, Public Health Agency of Canada, Winnipeg, Canada. ${ }^{9}$ Department of Laboratory Medicine and Pathobiology, University of Toronto, Toronto, Canada.

${ }^{10}$ Tropical Disease Unit, Toronto General Hospital, 200 Elizabeth Street, 13EN-218, Toronto, ON M5G 2C4, Canada.

Received: 31 August 2017 Accepted: 27 March 2018

Published online: 04 April 2018

\section{References}

1. Riley HD Jr. History of the cytomegalovirus. Southern Med J. 1997;90(2):84-90.

2. Goar EL, Potts CR. The relationship of rubella in the mother to congenital cataracts in the child. Trans Am Ophthalmol Soc. 1945;43:135-49.

3. Zika Working Group on behalf of the Committee to Advise on Tropical Medicine and Travel (CATMAT). Canadian recommendations on the prevention and treatment of Zika virus: update. Can Comm Dis Rep. 2016; 42:101-11.

4. Duffy MR, Chen TH, Hancock WT, et al. Zika virus outbreak on Yap Island, Federated States of Micronesia. N Engl J Med. 2009;360(24):2536-43.

5. Lanciotti RS, Kosoy OL, Laven JJ, et al. Genetic and serologic properties of Zika virus associated with an epidemic, yap state, Micronesia, 2007. Emerg Infect Dis. 2008;14(8):1232-9.

6. Balm MN, Lee CK, Lee HK, Chiu L, Koay ES, Tang JWA. Diagnostic polymerase chain reaction assay for Zika virus. J Med Virol. 2012;84(9):1501-5.

7. Honein MA, Dawson AL, Petersen EE, et al. Birth defects among fetuses and infants of US women with evidence of possible Zika virus infection during pregnancy. JAMA. 2017;317:59-68.

8. Cragan JD, Mai CT, Petersen EE, et al. Baseline prevalence of birth defects associated with congenital Zika virus infection - Massachusetts, North 
Carolina, and Atlanta, Georgia, 2013 - 2014. Morb Mortal Wkly Rep. 2017; 66(8):219-22.

9. Rasmussen SA, Jamieson DJ, Honein MA, Petersen LR. Zika virus and birth defects-reviewing the evidence for causality. N Engl J Med. 2016; 374(20):1981-7.

10. Franca GV, Schuler-Faccini L, Oliveira WK, et al. Congenital Zika virus syndrome in Brazil: a case series of the first 1501 livebirths with complete investigation. Lancet. 2016;388(10047):891-7.

11. Sarno M, Sacramento GA, Khouri R, et al. Zika virus infection and stillbirths: a case of Hydrops Fetalis, Hydranencephaly and fetal demise. PLoS Negl Trop Dis. 2016;10(2):e0004517.

12. Driggers RW, Ho CY, Korhonen EM, et al. Zika virus infection with prolonged maternal Viremia and fetal brain abnormalities. N Engl J Med. 2016;374(22): 2142-51.

13. Pomar $\mathrm{L}$, Malinger $\mathrm{G}$, Benoist $\mathrm{G}$, et al. Association between Zika virus and foetopathy: a prospective cohort study in French Guiana. Preliminary report. Ultrasound Obstet Gynecol. 2017:49(6):729-36.

14. Besnard M, Eyrolle-Guignot D, Guillemette-Artur P, et al. Congenital cerebral malformations and dysfunction in fetuses and newborns following the 2013 to 2014 Zika virus epidemic in French Polynesia. Euro Surveill. 2016;21(13) https://doi.org/10.2807/1560-7917.ES.2016.21.13.30181.

15. Carvalho FH, Cordeiro KM, Peixoto AB, et al. Associated ultrasonographic findings in fetuses with microcephaly because of suspected Zika virus (ZIKV) infection during pregnancy. Prenatal Diagn. 2016;36(9):882-7.

16. Mlakar J, Korva M, Tul N, et al. Zika virus associated with Microcephaly. N Engl J Med. 2016:374(10):951-8

17. Oliveira Melo AS, Malinger G, Ximenes R, Szejnfeld PO, Alves Sampaio S, Bispo de Filippis AM. Zika virus intrauterine infection causes fetal brain abnormality and microcephaly: tip of the iceberg? Ultrasound Obstet Gynecol. 2016;47(1):6-7.

18. Cortes MS, Rivera AM, Yepez M, et al. Clinical assessment and brain findings in a cohort of mothers, fetuses and infants infected with Zika virus. Am J Obstet Gynecol. 2018; https://doi.org/10.1016/..ajog.2018.01.012.

19. Shapiro-Mendoza CK, Rice ME, Galang RR, et al. Pregnancy outcomes of after maternal Zika virus infection during pregnancy - U.S. territories, January 1, 2016-April 25, 2017. Morb Mortal Wkly Rep. 2017;66(23):615-21.

20. Adhikari EH, Nelson DB, Johnson KA, et al. Infant outcomes among women with Zika virus infection during pregnancy: results of a large prenatal Zika screening program. Am J Obstet Gynecol. 2017;216(3):292. e1-292.e8

21. Suy A, Sulleiro E, Rodo C, et al. Prolonged Zika virus Viremia during pregnancy. N Engl J Med. 2016;375(26):2611-3.

22. Paz-Bailey G, Rosenberg ES, Doyle K, et al. Persistent of Zika virus in bodily fluids - preliminary report. N Engl J Med. 2017; https://doi.org/10.1056/ NEJMoa1613108

23. Bingham AM, Cone M, Mock V, et al. Comparison of test results for Zika virus RA in urine, serum, and saliva specimens from persons with travelassociated Zika virus disease - Florida, 2016. MMWR Morbid Mortal Wkly Rep. 2016;65(18):475-8.

24. Russell K. Update: interim guidance for the evaluation and management of infants with possible congenital Zika virus infection—United States, 2016. MMWR Morbid Mortal Wkly Rep. 2016;65:870-8.

25. Moore CA, Staples JE, Dobyns WB, et al. Characterizing the pattern of anomalies in congenital Zika syndrome for pediatric clinicians. JAMA Pediatr. 2016;171(3):288-95.

26. Barzon L, Pacenti M, Franchin E, et al. Infection dynamics in a traveller with persistent shedding of Zika virus RNA in semen for six months after returning from Haiti to Italy, January 2016. Euro Surveill. 2016;21(32). https:// doi.org/10.2807/1560-7917.ES.2016.21.32.30316

27. Oduyebo T, Igbinosa I, Petersen EE, et al. Update: interim guidance for health care providers caring for pregnant women with possible Zika virus exposure - United States, July 2016. MMWR Morbid Mortal Wkly Rep. 2016; 65(29):739-44

\section{Submit your next manuscript to BioMed Central and we will help you at every step:}

- We accept pre-submission inquiries

- Our selector tool helps you to find the most relevant journal

- We provide round the clock customer support

- Convenient online submission

- Thorough peer review

- Inclusion in PubMed and all major indexing services

- Maximum visibility for your research

Submit your manuscript at www.biomedcentral.com/submit

CBiomed Central 